\title{
COMBINING COUPLED SKOROKHOD SDES AND LATTICE GAS FRAMEWORKS FOR MULTI-FIDELITY MODELLING OF COMPLEX BEHAVIORAL SYSTEMS
}

\author{
Thi Kim Thoa Thieu*, Roderick Melnik*† \\ *M3AI Laboratory, MS2Discovery Interdisciplinary Research Institute \\ Wilfrid Laurier University, Waterloo, ON, Canada N2L 3C5 \\ ${ }^{\dagger}$ BCAM - Basque Center for Applied Mathematics, E-48009, Bilbao, Spain \\ Email: \{tthieu, rmelnik\}@wlu.ca
}

Key words: Behavioral Systems, Coupled Systems of SDEs, Uncertainties in Queueing Network Models, Kinetic Monte Carlo Methods, Skorokhod Equations, Modelling of Epidemics, Asymptomatic Hosts of SARS-CoV-2

\begin{abstract}
To model reliably behavioral systems with complex bio-social interactions, accounting for uncertainty quantification, is critical for many application areas. However, in terms of the mathematical formulation of the corresponding problems, one of the major challenges is coming from the fact that corresponding stochastic processes should, in most cases, be considered in bounded domains, possibly with obstacles. This has been known for a long time and yet, very little has been done for the quantification of uncertainties in modelling complex behavioral systems described by such stochastic processes. In this paper, we address this challenge by considering a coupled system of Skorokhodtype stochastic differential equations (SDEs) describing interactions between active and passive participants of a mixed-population group. In developing a multi-fidelity modelling methodology for such behavioral systems, we combine low- and high-fidelity results obtained from (a) the solution of the underlying coupled system of SDEs and (b) simulations with a statistical-mechanics-based lattice gas model, where we employ a kinetic Monte Carlo procedure. Furthermore, we provide representative numerical examples of healthcare systems, subject to an epidemic, where the main focus in our considerations is given to an interacting particle system of asymptomatic and susceptible populations.
\end{abstract}

\section{Introduction}

The modelling of complex behavioral systems with bio-social interactions offers many challenging questions to science and technology in general. In developing more realistic 
models, the uncertainty quantification plays an important role for the setting of mathematical formulation of the problems. Uncertainties coming from different sources are ubiquitous in the modelling of behavioral systems with bio-social interactions [1]. In terms of the mathematical formulation of the corresponding problems, one of the major challenges is coming from the fact that corresponding stochastic processes describing such systems should, in most cases, be considered in bounded domains, possibly with obstacles $[2,3,4]$. Hence, for the class of stochastic models with bounded domains, the appropriate boundary conditions must be specified. The boundary conditions can be, for example, reflecting, absorbing or even mixed boundary conditions. Recent developments reported in $[5,7]$ bring us to the study of a system of Skorokhod-type SDEs modelling the interactions between active and passive populations. Many important operations research problems, including those arising in queueing theory, require the models describing reflecting stochastic dynamics. Moreover, this class of queueing theory models is important in many applied fields, including the emergency medical aid systems [6], passenger services in air terminals, road traffic and intelligent transportation, as well as various types of other systems and processes, represented as some operations on material, informational or other flows. On the other hand, the topics of SARS-CoV-2, which is an ongoing global pandemic, have recently attracted the interest of the scientific community. One of the

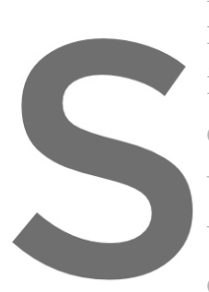
main challenges lies ics of social factors
can help prevent the
which can cause a n
vant results are avail complex systems in gotro
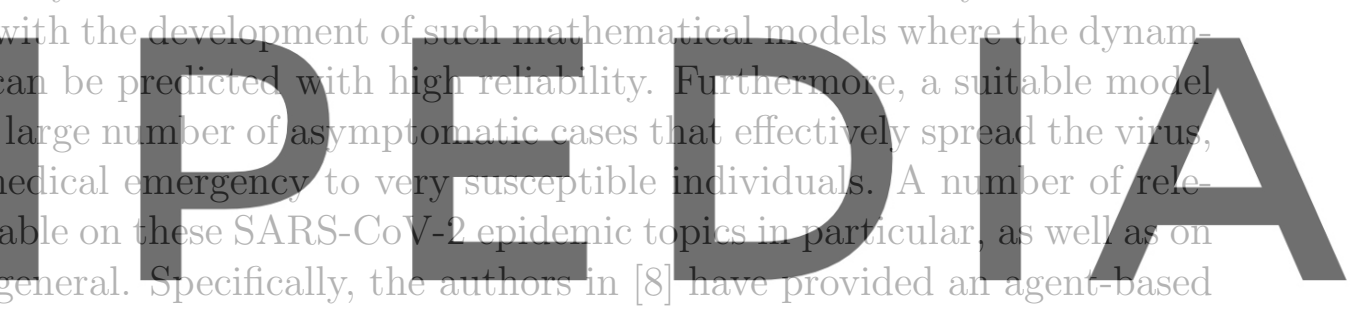
framework to model self-organizing systems at different levels of organization, physical,

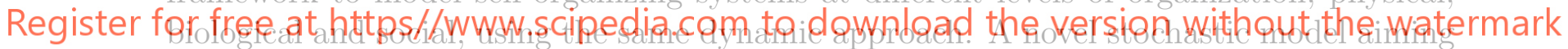

to account for the unique transmission dynamics of COVID-19 and capture the effects of

intervention measures has been proposed in [9]. An agent-based model for simulations of infection transmission through bio-social stochastic dynamics has been investigated in [10]. The same authors also extended this agent-based modelling framework to shed light on the role of asymptomatic hosts and unravel the interplay between the biological and social factors of these nonlinear stochastic processes at high temporal resolution in [11]. In [12], the authors have considered a model for quantifying asymptomatic infection and transmission of COVID-19 by using observed cases, serology, and testing capacity. The presence of asymptomatic hosts in the population is a major challenge for controlling the epidemics given the fact that a new host can contract the virus with a delay. The asymptomatic virus carriers can infect a susceptible individual, who, depending on the susceptibility, may or may not develop symptoms. Similarly, the viruses produced by presymptomatic hosts can lead to asymptomatic as well as symptomatic cases. Taking the inspiration from the study of a system of Skorokhod-type SDEs together with applications in epidemic problems, our representative example here is pertinent to the modelling of 
healthcare systems, subject to an epidemic, where we have to deal with distinct behaviors caused by the interactions between asymptomatic and susceptible populations.

Therefore, in what follows, we consider a system of SDEs of Skorohod type aimed at modelling the dynamics of active-passive populations. In particular, we show that a model of queueing theory converges to a system of reflected SDEs via a limit theorem. Furthermore, we develop a multi-fidelity modelling methodology (e.g. in [13, 14]) for our behavioral system. The analysis of this model is carried out by combining low- and high-fidelity results obtained from the solution of the underlying coupled system of SDEs and from the simulations with a statistical-mechanics-based lattice gas model, where we employ a kinetic Monte Carlo procedure. We provide details of the models developed here, as well as several representative numerical examples of healthcare systems, subject to an epidemic, where we consider an interacting particle system of asymptomatic and susceptible populations.

\section{Model description}

In this section, we consider a general queueing theory model for active and passive particles. The geometry is a square lattice $\Lambda:=\{1, \ldots, L\} \times\{1, \ldots, L\} \subset \mathbb{Z}^{2}$ of side length $L$, where $L$ is an odd positive integer number. $\Lambda$ will be referred in this context

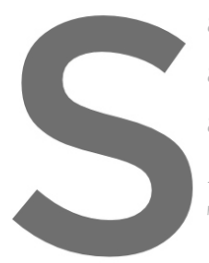
as room. An elemen

are said nearest neight as a set made of $\omega$ pat

L, located on the top

This mimies the preser

will be called width of the exit. In addition.
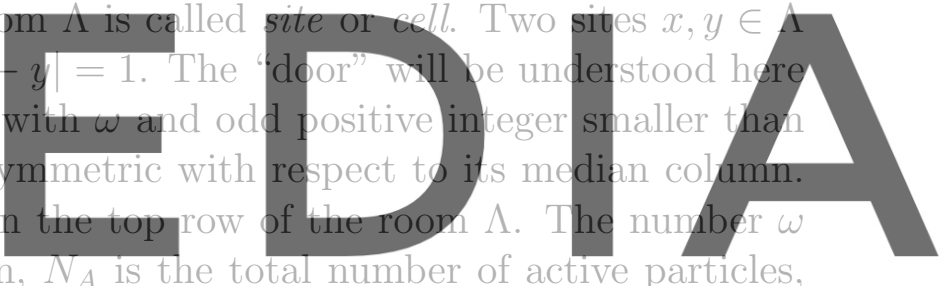

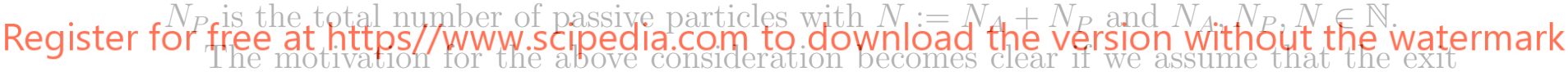

door is a service station located on the top row of the geometry, and there are $\omega$ service gates at the service station. Every active particle's target is to reach these service gates and to leave the geometry immediately after that, while the passive particles cannot leave the room due to invalid access to the service gates.

We introduce a special combination of SDEs with jumps (see, e.g., [15]) for the following active-passive populations.

\subsection{Active population}

For $i \in\left\{1, \ldots, N_{A}\right\}$ and $t \in[0, T]$, let $X_{A_{i}}$ denote the position of particle $i$ belonging to the active population at time $t$. Suppose that $f_{A_{i}}:[0, T] \times \mathbb{R}^{2} \longrightarrow \mathbb{R}^{2}, g_{A_{i}}:[0, T] \times \mathbb{R}^{2} \longrightarrow$ $\mathbb{R}^{2} \times \mathbb{R}^{2}$. We consider the dynamics of active particles that is governed by

$$
\left\{\begin{array}{l}
d X_{A_{i}}(t)=f_{A_{i}}\left(X_{A_{i}}(t), X_{P_{k}}(t), t\right)+g_{A_{i}}\left(X_{A_{i}}(t), X_{P_{k}}(t), t\right) d W_{A_{i}}(t)+d \Phi_{A_{i}}(t), \\
X_{A_{i}}(0)=X_{A_{i 0}} .
\end{array}\right.
$$


In other words, the active population of the system is described by the following process:

$$
\begin{aligned}
X_{A_{i}}^{\alpha}(t) & =X_{A_{i}}^{\alpha}(0)+\int_{0}^{t} f_{A_{i}}\left(X_{A_{i}}^{\alpha}(s), X_{P_{k}}^{\mu}(s), s\right) d s \\
& +\int_{0}^{t} g_{A_{i}}\left(X_{A_{i}}^{\alpha}(s), X_{P_{k}}^{\mu}(s), s\right) d W_{A_{i}}(s)+\Phi_{A_{i}}^{\alpha}(t) \\
& =X_{A_{i}}^{\alpha}(0)+Y_{A_{i}}^{\alpha}(t)+\Phi_{A_{i}}^{\alpha}(t),
\end{aligned}
$$

where

$$
Y_{A_{i}}^{\alpha}(t):=\int_{0}^{t} f_{A_{i}}\left(X_{A_{i}}^{\alpha}(s), X_{P_{k}}^{\mu}(s), s\right) d s+\int_{0}^{t} g_{A_{i}}\left(X_{A_{i}}^{\alpha}(s), X_{P_{k}}^{\mu}(s), s\right) d W_{A_{i}}(s) .
$$

Here, $\left(W_{A_{i}}\right)_{i}$ is a sequence of independent standard Brownian motions, while $Y_{A_{i}}^{\alpha}$ can be interpreted as the arrival times of active particles, and $\Phi_{A_{i}}^{\alpha}(t)$ is the cumulative lost service capacity over $[0, T]$.

\subsection{Passive population}

For $k \in\left\{1, \ldots, N_{P}\right\}$ and $t \in[0, T]$, let $X_{P_{k}}$ denote the position of particle $k$ belonging at

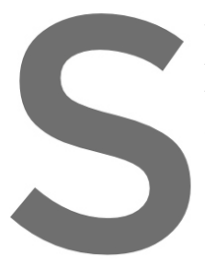

time $t$ to the passive

$\mathbb{R}^{2} \times \mathbb{R}^{2}$. The dynanic

differential equations
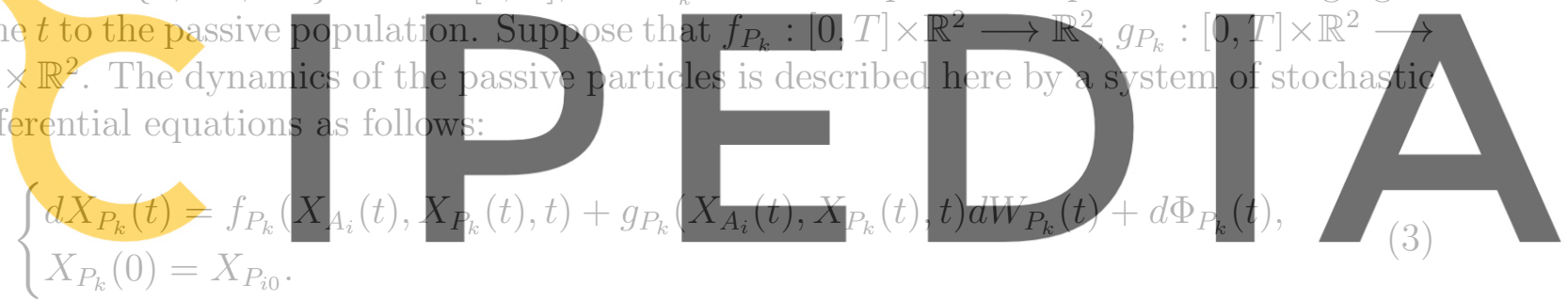

Register for free at https//www.scipedia.com to download the version without the watermark

in other words, the passive population of the system is described by the following process:

$$
\begin{aligned}
X_{P_{k}}^{\mu}(t) & =X_{P_{k}}^{\mu}(0)+\int_{0}^{t} f_{P_{k}}\left(X_{A_{i}}^{\alpha}(s), X_{P_{k}}^{\mu}(s), s\right) d s \\
& +\int_{0}^{t} g_{P_{k}}\left(X_{A_{i}}^{\alpha}(s), X_{P_{k}}^{\mu}(s), s\right) d W_{P_{k}}(s)+\Phi_{P_{k}}^{\mu}(t) \\
& =X_{P_{j}}^{\mu}(0)+Y_{P_{j}}^{\mu}(t)+\Phi_{P_{j}}^{\mu}(t),
\end{aligned}
$$

where

$$
Y_{P_{j}}^{\mu}(t):=\int_{0}^{t} f_{P_{k}}\left(X_{A_{i}}^{\alpha}(s), X_{P_{k}}^{\mu}(s), s\right) d s+\int_{0}^{t} g_{P_{k}}\left(X_{A_{i}}^{\alpha}(s), X_{P_{k}}^{\mu}(s), s\right) d W_{P_{k}}(s) .
$$

Here, $\left(W_{P_{k}}\right)_{k}$ is a sequence of independent standard Brownian motions. Similarly, $Y_{P_{k}}^{\mu}$ can be interpreted as the arrival times of passive particles, and $\Phi_{P_{k}}^{\mu}(t)$ is the cumulative lost service capacity over $[0, T]$. 


\subsection{Coupling mechanisms between active and passive populations}

The drift coefficients $f_{A_{i}}$ and $f_{P_{k}}$ in (1) and (3) can be considered as functions describing the interactions between active and passive particles. The specific formulations of $f_{A_{i}}$ and $f_{P_{k}}$ can be defined differentially in various models (see, e.g., $[5,16]$ ). Similarly, the detailed formulation of diffusion terms $g_{A_{i}}$ and $g_{P_{k}}$ in (1) and (3) are defined depending on different models.

This behavioral system in the context of operations research applications can be viewed as an $M / M / \omega / N$ queueing system with the following assumptions:

a) there are $N$ particles that request services (for example, healthcare systems),

b) the maximum number of requests in the system equals $\omega$,

c) the arrival times of active and passive particles are exponential i.i.d random variables with intensities $\alpha$ and $\mu$, respectively.

Note that the notation $M / M / \omega / N$ means that the queueing model contains $\omega$ service points and $N$ places for waiting, while $M$ represents Poisson (or another probabilistic) arrival process (i.e., exponential inter-arrival times see, e.g., [17]).
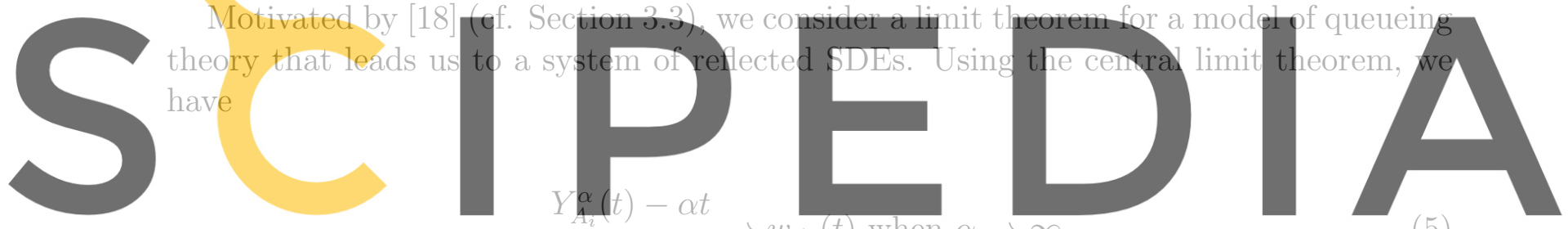

(5)

Register for free at https//www.scipedia.com to download the version without the watermark and

$$
\frac{Y_{P_{k}}^{\mu}(t)-\mu t}{\sqrt{\mu}} \rightarrow w_{P_{k}}(t) \text { when } \mu \rightarrow \infty
$$

in $\Lambda$. Similarly, we also have

$$
\left(\frac{X_{A_{i}}^{\alpha}(t)}{\sqrt{\alpha}}, \frac{\Phi_{A_{i}}^{\alpha}(t)}{\sqrt{\alpha}}\right) \rightarrow\left(\xi_{A_{i}}(t), \phi_{A_{i}}(t)\right) \text { when } \alpha \rightarrow \infty
$$

and

$$
\left(\frac{X_{P_{k}}^{\mu}(t)}{\sqrt{\mu}}, \frac{\Phi_{P_{k}}^{\mu}(t)}{\sqrt{\mu}}\right) \rightarrow\left(\xi_{P_{k}}(t), \phi_{P_{k}}(t)\right) \text { when } \mu \rightarrow \infty
$$

in $\Lambda$. 
As a result, the pairs $\left(\xi_{A_{i}}(t), \phi_{A_{i}}(t)\right)$ and $\left(\xi_{P_{k}}(t), \phi_{P_{k}}(t)\right)$ are the solutions of the following Skorokhod type equations:

$$
\begin{aligned}
& \xi_{A_{i}}(t)=w_{A_{i}}(t)+\phi_{A_{i}}(t), \\
& \xi_{P_{k}}(t)=w_{P_{k}}(t)+\phi_{P_{k}}(t) .
\end{aligned}
$$

The reflecting stochastic queueing theory model we discussed here is a low-fidelity modelling methodology for general behavioral systems. In order to approximate this reflecting stochastic dynamics problem, we are going to combine the coupled system of SDEs with a high-fidelity modelling method by using a statistical-mechanics-based lattice gas approach. We provide more detailed descriptions of the lattice gas approximation by showing numerical examples in the next section.

\section{Numerical examples}

In this section we consider an application of our general queueing theory model in a healthcare system. In particular, we investigate an interacting particles system of active and passive populations on the example of modelling the interactions of asymptomatic

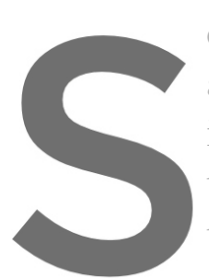
carriers and a susceptible population. We assune that active particles can be seen as
asymptomatic hosts (carriers) while passive particles can be considered as susceptible
individuals. The process is described as follows: the asymptomatic hosts do not know that
they carry the virus and unknowingly spread the virus to the susceptible individuals. After
that, the whole infected population may have severe symptoms and may need intensive
care. Since the asymptomatic groups have not had any pronounced symptoms yet, the
infectious dose of viable SARS-CoV-2 virus that needs to be present in the body to cause Register forf free at https/drwww scipedia.com to download the version without the watermark

individual will be infected provided that individual communicates with asymptomatic carriers at his nearest neighbor sites.

The representative examples we consider here for two different species of active and passive particles, moving inside lattice $\Lambda$ defined as in the previous section (we use in the notation the symbols A and P to respectively refer to them). Similarly, there are $\omega$ service gates at the service station and these service gates are located on the top row of the room. Note that the sites of the external boundary of the room, i.e. the sites $x \in \mathbb{Z}^{2} \backslash \Lambda$, are such that there exists $y \in \Lambda$ nearest neighbor of $x$ which cannot be accessed by the particles. We call the state of the system configuration $\eta \in \Omega=\{-1,0,1\}^{\Lambda}$ and we shall say that the site $x$ is empty if $\eta_{x}=0$, occupied by an active particle if $\eta_{x}=1$, and occupied by a passive particle if $\eta_{x}=-1$. The number of active (respectively, passive) particles in the configuration $\eta$ is given by $n_{\mathrm{A}}(\eta)=\sum_{x \in \Lambda} \delta_{1, \eta_{x}}\left(\operatorname{resp} . n_{\mathrm{P}}(\eta)=\sum_{x \in \Lambda} \delta_{-1, \eta_{x}}\right)$, where $\delta_{\text {, }}$, is Kronecker's symbol. Their sum is the total number of particles in the configuration $\eta$.

The original idea of this population dynamics has been proposed in [19, 21] in a lattice gas framework. Taking the inspiration from this stochastic dynamics in [19], we model 


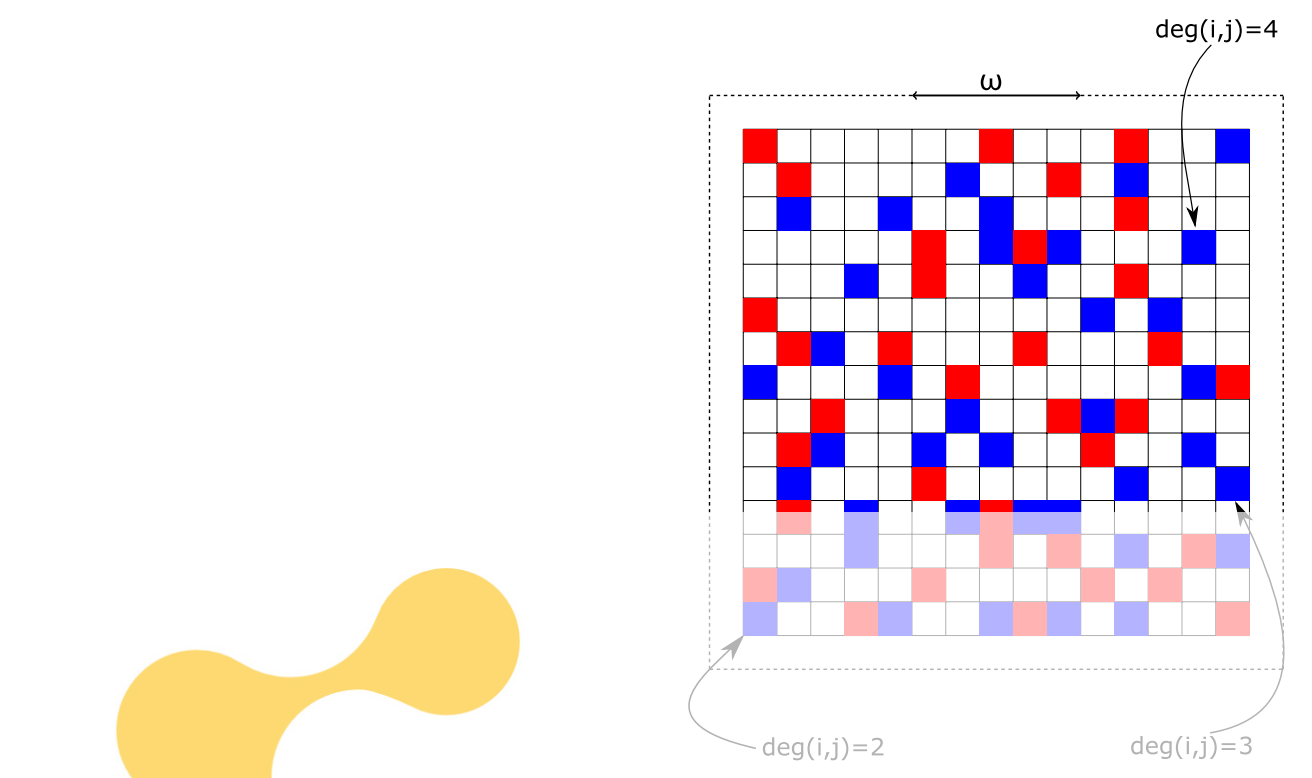

Figure 1: (Color online) Schematic representation of our lattice model. Blue and red squares denote passive and active particles, respectively, while the white squares within the geometry represents the empty spots. The thick dashed line surrownding a large fra ti reflecting boundary conditions. equal to $\omega$.

system since they need intensive care units. In general, most people who are infected with the COVID-19 virus will get mild to moderate respiratory illness and recover without requiring special treatment. Older individuals and also those groups of people of any age who have other serious health problems like cardiovascular disease, diabetes, chronic respiratory disease, and cancer are more likely to develop serious illness. We assume that the drift quantity $\varepsilon>0$ is the level of serious illness patients who need intensive cares. In particular, the larger $\varepsilon$ of active particles the more patients need intensive care units (the more load will be placed on the intensive care unit). Note that our dynamics are incorporated in the continuous time Markov chain $\eta(t)$ on $\Omega$ with rates $c\left(\eta, \eta^{\prime}\right)$, for the detailed definitions of the rates $c(\eta(t), \eta)$, see, e.g., $[19,20]$.

In this section, the numerical results are obtained by using the kinetic Monte Carlo (KMC) method. In particular, we simulate the presented model by using the following scheme: we extract at time $t$ an exponential random time $\tau$ as a function with parameter which is the total rate $\sum_{\zeta \in \Omega} c(\eta(t), \zeta)$ of exchanging between configurations (e.g. in [19]), 
then set the time $t$ equal to $t+\tau$. Next, we select a configuration using the probability distribution $c(\eta(t), \eta) / \sum_{\zeta \in \Omega} c(\eta(t), \zeta)$ and set $\eta(t+\tau)=\eta$ (for the detailed definitions of the rates $c(\eta(t), \eta)$, see e.g. $[19,20])$.

This numerical scheme has been studied in [19], where the authors implemented a version of KMC in the context of a lattice gas model with two species of particles. Further validation of the numerical methodology used here was reported in [20], based on a counterflow crowd dynamics model. The overall dynamics of our system is based on a continuous time Markov chain, i.e. the process will change state according to an exponential random variable and then move to a different state as specified by the probabilities of a stochastic matrix, together with a simple exclusion process. On the other hand, we use a statistical-mechanics-based lattice gas framework where we employ a kinetic Monte Carlo procedure to supplement the methodology described in the previous section. Furthermore, in general, Monte Carlo statistical methods, particularly those based on Markov chains, are one way to sample the distribution of the input random variable. Hence, the methodology used in our model can be considered as a multi-fidelity approach to statistical inference (e.g. in [14, 22]).

In the current version, we have improved the original simulations provided in $[19,20]$ by creating the interaction among active and passive particles. We have each site on the lattice connected site $(i, j)$ will be calle
is described as follows
particle, then we coun
total number of active
the passive particle

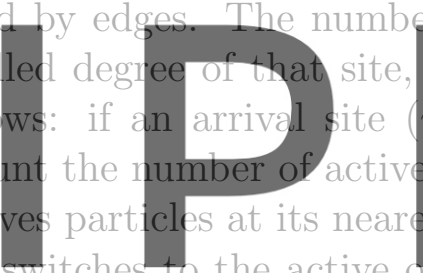
tches to the active
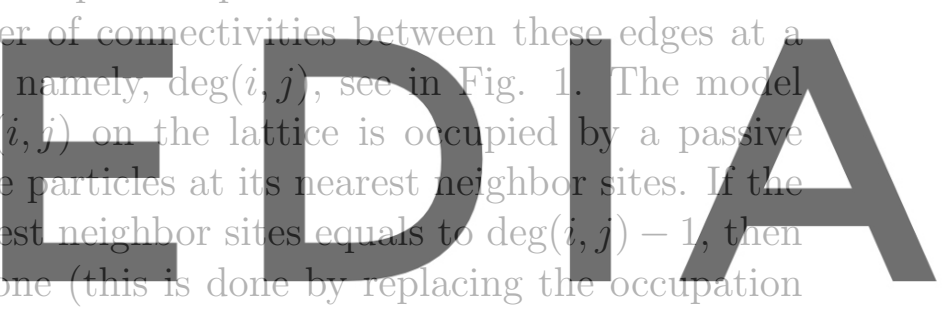

number of the passive particle at its site equal to 0 , at the same time we let the occupation

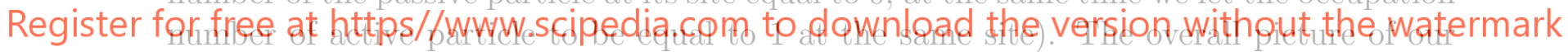

stochastic dynamics is illustrated in Fig. 2, where we show the configuration of the system at different times.

In the simulations, we fix the parameters for $L=30, \omega=20$ and $N_{A}=N_{P}=1200$. The main numerical results of our analysis are shown in Fig. 3, where we have plotted the total number of infected individuals and the infected individuals currents as functions of time, for different values of $\varepsilon$. The current is defined in the infinite time limit by the ratio between the total number of infected individuals, that in the interval $(0, t)$ passed through the service gates to enter the healthcare system, and the time $t$ (see e.g. in [21]). Our numerical results have demonstrated that the latent infection transmissions caused by asymptomatic carriers can increase the needed intensive care. In Fig. 3, the virus carriers increase over time. This is visible in all of the cases of $\varepsilon$. In the left panel of Fig. 3, when we increase the values of $\varepsilon$ from 0 to 0.3 , the currents of infected individuals increase. In particular, the current of virus carriers who need intensive care in the case of $\varepsilon=0$ is smaller than in the case of $\varepsilon=0.05$. Similarly, the current of virus hosts in the case of $\varepsilon=0.05$ is smaller than in the case of $\varepsilon=0.1$, and etc. When the virus carriers do 

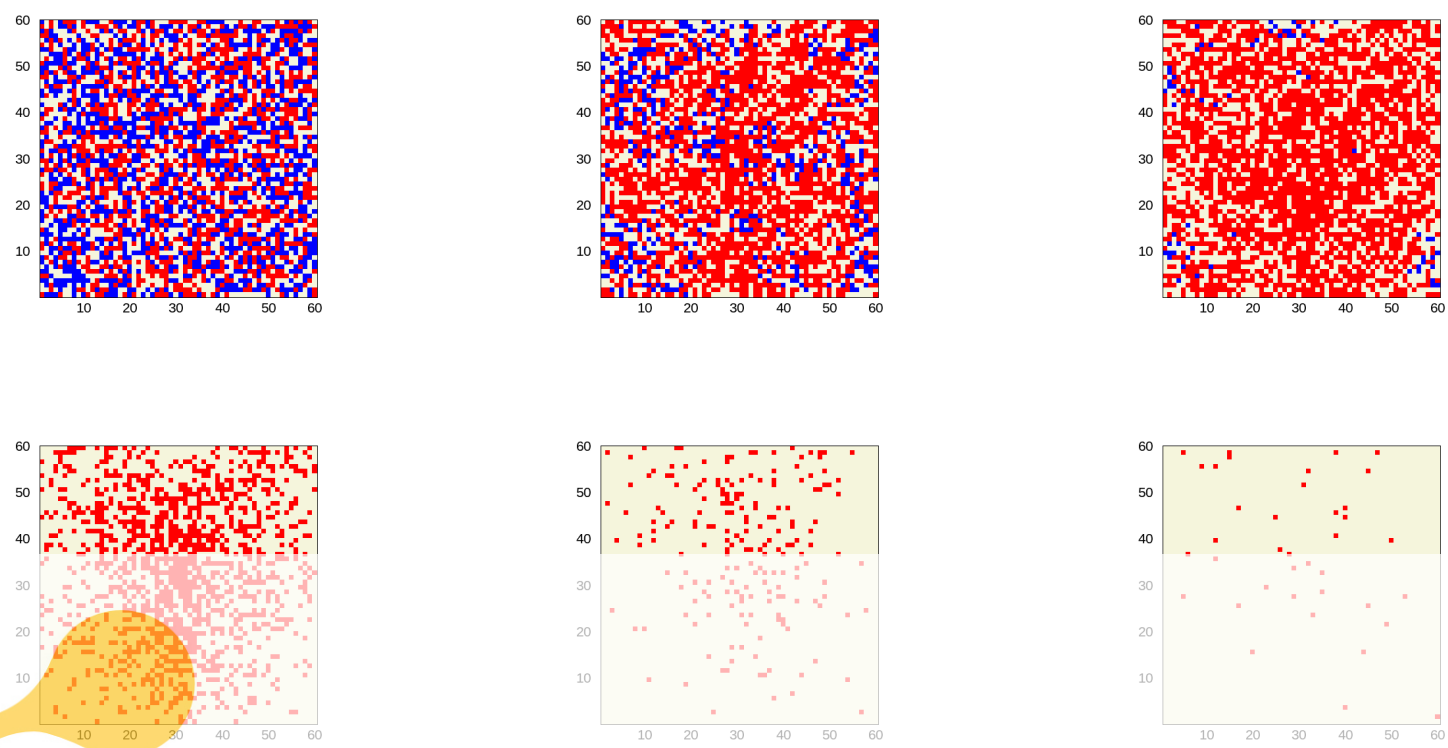

Figure 2: (Color online) The configurations of the model at different times (increasing in lexico-

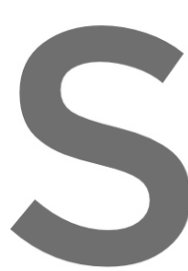
graphic order). Parame

blue pixels denote passi left panel) there are 120

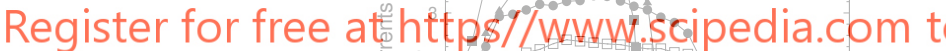
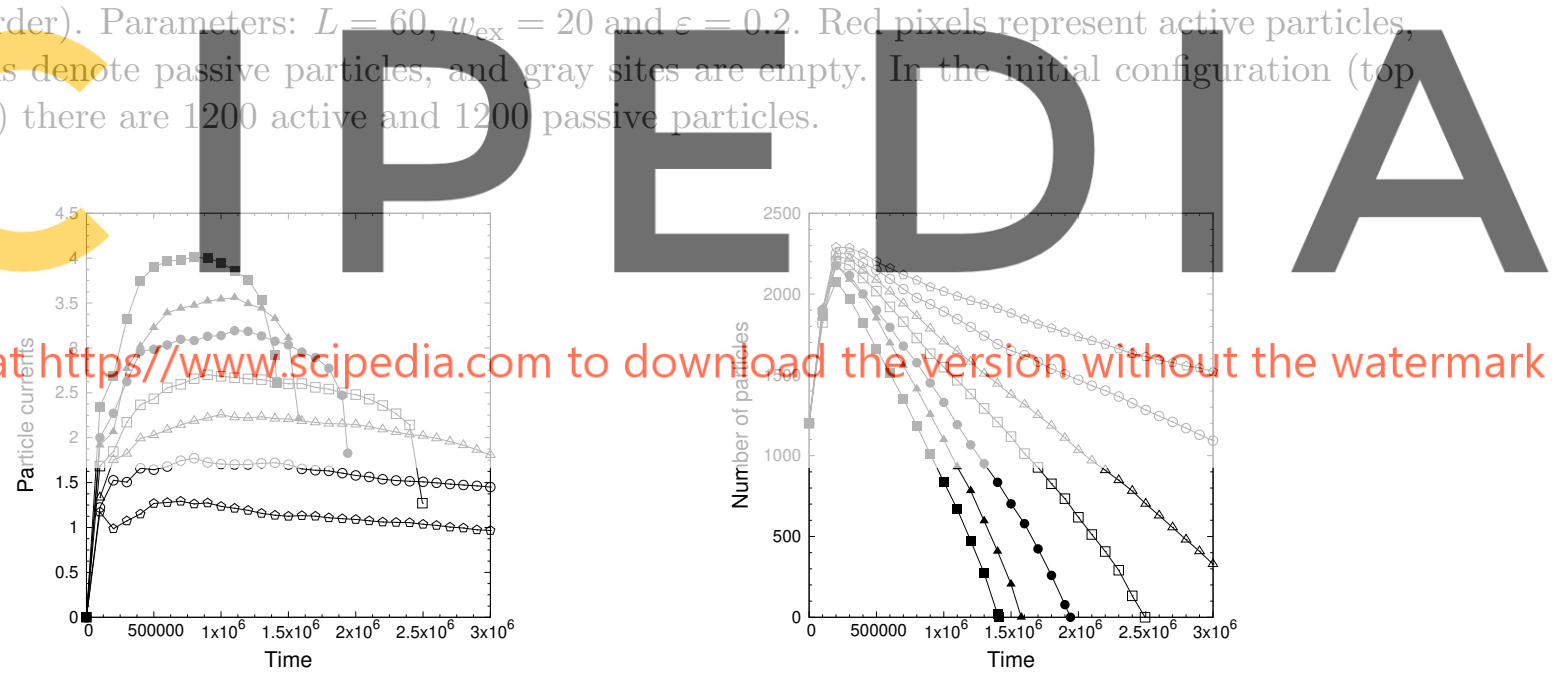

Figure 3: Left panel: Evolution of the infected individual currents as a function of time for $L=60$, $N_{A}=N_{P}=1200, \varepsilon=0$ (empty pentagons), $\varepsilon=0.05$ (empty circles), $\varepsilon=0.1$ (empty triangles), $\varepsilon=0.15$ (empty squares), $\varepsilon=0.2$ (solid circles), $\varepsilon=0.25$ (solid triangles) and $\varepsilon=0.3$ (solid squares). Right panel: Behavioral pattern of the infected individuals for $L=60, N_{A}=N_{P}=1200, \varepsilon=0$ (empty pentagons), $\varepsilon=0.05$ (empty circles), $\varepsilon=0.1$ (empty triangles), $\varepsilon=0.15$ (empty squares), $\varepsilon=0.2$ (solid circles), $\varepsilon=0.25$ (solid triangles) and $\varepsilon=0.3$ (solid squares).

not need intensive cares, this means that $\varepsilon=0$. This is due to the fact that the infected individuals may or may not develop symptoms, or even have slight symptoms. The right panel of Fig. 3 shows the corresponding behavior of the total number of particles. It 
is clear that the number of virus carriers increases over time up to some value where it attains a maximum (at $N=2400$ ). However, soon after the number of virus hosts reaches its maximum, the number of infected individuals is reduced over time since they already entered the healthcare system by passing through the healthcare service gates. Furthermore, when $\varepsilon$ increases, there is an increase in the needed intensive care units of virus carriers. It is clear that if the asymptomatic populations spread the virus in the society, the virus carriers will significantly increase after short time. This effect is visible in the right panel of Fig. 3, from $t=0$ to $t \approx 300000$ ( $t$ represents the Monte Carlo time steps), the number of infected individuals dramatically increases from 1200 to around 2300. For example, assuming that the intensive care units capacity is 1200, the hospital would not have enough beds for intensive care patients. Finally, it is worth noting that the effect of asymptomatic hosts can cause high pressure on the healthcare systems. To prevent this situation, the social lockdown and/or vaccination will need to be considered and built into the modelling scenarios.

\section{Concluding remarks}

We have presented conceptually a procedure for combining coupled Skorokhod SDEs and a lattice gas framework for multi-fidelity modelling of complex behavioral systems. In particular, we have studied a cotpled system of Skoroknod-type SDEs, modelling th interactions between active and passive populations. We have discussed the relationship
between a general queueing theory model and a system of reflected SDEs via a limpt
theorem. Using a multi-fidelity approach to statistical inference, we have considerdd an
application of our reflected stochastic dynamies in the healtheare system, subjected to an epidemic, where the main focus in our analysis has been given to the interactions between asymptomatic and susceptible populations in a statistical-mechanics-based lattice for free at https// wwW. scipedia.com to download the version without the watermark gas framework? Based on our numerical experiments, we have observed that the virus spreading of asymptomatic hosts affects the healthcare system by an increase of intensive care units. The overall dynamics of our model is based on a continuous time Markov chain together with a simple exclusion process. In general, Monte Carlo methods, particularly those based on Markov chains, are now an essential component of the standard set of techniques used by quantitative researchers such as computational biologists, probabilists, and statisticians. In fact, the infection transmission of asymptomatic population is hard to measure. Our multi-fidelity model has given a prediction on how the infection transmission of asymptomatic carriers behaves so that we can have suitable solutions to prevent the lack of intensive care units. In the current consideration, we have considered only the simplest case of the influence of asymptomatic carriers in the system. It would be instructive to analyze and compare our current model with extensions of the model accounting for lockdowns and/or vaccination scenarios. Also, the comparison between the theoretical prediction of our model with the real data would need to be investigated further. Furthermore, the ideas presented in this contribution may be extended to other fields such as crowd dynamics and dynamics of neurons in nervous systems. 


\section{Acknowledgment}

Authors are grateful to the NSERC and the CRC Program for their support. RM is also acknowledging support of the BERC 2018-2021 program and Spanish Ministry of Science, Innovation and Universities through the Agencia Estatal de Investigacion (AEI) BCAM Severo Ochoa excellence accreditation SEV-2017-0718 and the Basque Government fund AI in BCAM EXP. 2019/00432. Both authors are acknowledging useful insight provided by Dr. Pilipenko, and TKTT thanks Dr. A. Muntean (Karlstad, Sweden), Dr. E.N.M. Cirillo (Rome, Italy) and Dr. M. Colangeli (L'Aquila, Italy) for very fruitful discussions on the topic of active-passive populations dynamics.

\section{REFERENCES}

[1] Bellomo, N., Bellouquid, A., Gibelli, L. and Outada, N. Mathematical models of crowd dynamics in complex venues. Springer (2017):119-160.

[2] Skorohod, A.V. Stochastic equations for diffusion process in a bounded domain. Theory of Probability and Its Applications. (1961) VI:264-274.

[3] Zhang, T. Lattice approximations of reflected stochastic partial differential equations driven by spacetime white noise. The Annals of Applied Probability. (2016) 26:36023629 .

[4] Buisson, J.D. and Touchette, H. Dynamical large deviations of reflected diffusions. Physical Review E. (2019) 17:460-477.

[5] Thieu, T.K.T. and Muntean, A. Well-posedness of a coupled system of Skorohod-like stochastic differential equations. Submitted (arXiv:2006.00232). (2020).

[6] Meares, H.D. and Jones, M.P. When a system breaks: queueing theory model of intensive care bed needs during the COVID-19 pandemic. Med. J. Aust. (2019) 212:470-471.

[7] Briand, P., Grannoum, A., Labart, C. Mean reflected stochastic differential equations with jumps. Advances in Applied Probability. (2020) 52:523-562.

[8] Schweitzer, F. An agent-based framework of active matter with applications in biological and social systems. European Journal of Physics. (2019) 40:014003.

[9] Zhang, Y., You, C., Cai, Z., Sun, J., Hu, W. and Zhou, Z.H. Prediction of the COVID-19 outbreak in China based on a new stochastic dynamic model. Scientific Reports. (2020) 10:21522.

[10] Tadíc, B. and Melnik, R. Modeling latent infection transmissions through biosocial stochastic dynamics. PLoS ONE. (2020) 15:e0241163. 
[11] Tadíc, B. and Melnik, R. Microscopic dynamics modeling unravels the role of asymptomatic virus carriers in SARS-CoV-2 epidemics at the interplay between biological and social factors. Computers in Biology and Medicine. (2021) 133:104422.

[12] Subramanian, R., He, Q. and Pascual, M. Quantifying asymptomatic infection and transmission of COVID-19 in New York City using observed cases, serology, and testing capacity. PNAS. (2021) 118:e2019716118.

[13] Peherstorfer, B., Kramer, B. and Willcox, K. Combining multiple surrogate models to accelerate failure probability estimation with expensive high-fidelity models. Journal of Computational Physics (2017) 341:61-75.

[14] Peherstorfer, B., Willcox, K. and Gunzburger, M. Survey of multifidelity methods in uncertainty propagation, inference, and optimization. SIAM Review. (2018) 60:550591.

[15] Situ, R. Theory of Stochastic Differential Equations with Jumps and Applications: Mathematical and Analytical Techniques with Applications to Engineering. Springer (2005).

[16] Philipowski, R. Interacting diffusions approximating the porous medium equation and propagation of chaos. Stochastic Processes and their Applications. (2018) 117:526-538.

[17] Kalashnikov, V.V. Mathematical Methods in Queuing Theory. Springer Science Business Media Dordrecht (1989).

[18] Pilipenko, A. An Introduction to Stochastic Differential Equations with Reflection. Lectures in Pure and Applied Mathematics. Institutional Repository of the University of Potsdam, Germany (2014).

[19] Cirillo, E.N.M., Colangeli, M., Muntean, A. and Thieu, T.K.T. A lattice model for active-passive pedestrian dynamics: a quest for drafting effects. Mathematical Biosciences and Engineering. (2019) 17:460-477.

[20] Cirillo, E.N.M., Colangeli, M., Muntean, A. and Thieu, T.K.T. When diffusion faces drift: consequences of exclusion processes for bi-directional pedestrian flows. Physica D. (2020) 413:132651.

[21] Colangeli, M., Muntean, A., Richardson, O., and Thieu, T.K.T. Modelling interactions between active and passive agents moving through heterogeneous environments. in G. Libelli, N. Bellomo (Eds), Crowd Dynamics, Modeling and Simulation in Science, Engineering and Technology, Boston, Birkhauser, Springer. (2019) 1:211-254.

[22] Robert, C., and Casella, G. Monte Carlo Statistical Methods. Springer (2004). 